\title{
A Fuzzy Environment Inventory Model with Partial Backlogging under Learning Effect
}

\author{
Isha Sangal \\ Centre for mathematical \\ sciences \\ Banasthali University, \\ Rajasthan
}

\author{
Anchal Agarwal \\ Centre for mathematical \\ sciences \\ Banasthali University, \\ Rajasthan
}

\author{
Smita Rani \\ Centre for mathematical \\ sciences \\ Dr. A.P.J Abdul Kalam \\ University, Lucknow
}

\begin{abstract}
In this article we developed an inventory model for noninstantaneous decaying items is considered under crisp and fuzzy environment. In this study we have considered stock dependent demand rate and variable deterioration. It is supposed that shortages are allowed and partially backlogged with exponential backlogging rate. Holding cost follows the learning curve. The deterioration rate, ordering cost, shortage cost and deterioration cost are assumed as a triangular fuzzy numbers. The aim of our study is to defuzzify the total cost function by signed distance method. This model is developed in both crisp and fuzzy surroundings. A numerical experiment is given to demonstrate the developed crisp and fuzzy models. Sensitivity analysis is implemented to examine the effect of parameters. The convexity of the total cost function is shown by graphically.
\end{abstract}

\section{Keywords}

Non-instantaneous-deterioration, Triangular fuzzy numbers, Signed distance, Learning, Partial backlogging

\section{INTRODUCTION}

Inventory optimization deals with the decision to minimize the total average cost or maximize the total average profit. To do this, the approach is to construct a mathematical model of the real life inventory system by taking into account various assumptions and approximations. In most of the inventory models, it is assumed that various parameters like demand rate and ordering cost, etc. are precisely known. But in reality, the nature of these parameters is uncertain, so it is important to consider them as fuzzy numbers. Considering the fuzzy set theory in inventory model brings authenticity to the model since fuzziness is the closest possible approach to reality. By considering approximations, fuzzy theory helps one to incorporate uncertainties in the model formulation making it closer to reality.

The concept of fuzzy set theory modeling was developed by Zadeh (1965). Jain (1976) deliberated a fuzzy inventory model on decision making in the presence of fuzzy variables. Some operations on fuzzy numbers was defined by Dubois and Prade (1978). A long term inventory policy making through fuzzy decisions was formulated by Kacpryzk and Staniewski (1982). Zimmerman (1983) applied to use fuzzy sets in operational research.

Demand is directly co-related to stock and cost. In a fuzzy environment, data of stock are not clear. So to take care demand \& inventory cost, it is important to avoid shortage. There are lots of factor, keeping open eyes on them, we can minimize fuzzy environment. Fuzzy inventory with backorder for fuzzy order quantity was investigated by Yao and Lee(1996). A Single Period Inventory Model with Fuzzy Demand was proposed by Kao and Hsu(2002). An inventory model with total demand and storing cost as triangular fuzzy numbers was developed by Yao and Chiang (2003). They applied the defuzzification by centroid method and signed distance method both. A multi-item, multi-objective inventory model for deteriorating items with stock- and time-dependent demand rate over a finite time horizon in fuzzy stochastic environment was presented by Mahapatra and Maiti (2006). Halim et. al. (2008) deliberated by an EOQ model for perishable items with stochastic demand and partial backlogging. A fuzzy inventory model without shortages by using triangular fuzzy number was presented by De and Rawat(2011). Some recent work in this direction is done by Jaggi et. al. (2012), Saha and Chakrabarti (2012), Dutta and Kumar (2013) and Kumar and Rajput (2015) etc.

Stock maintain has directly related to deterioration specially for perishable items and short expiry period goods. To control these we have to convert larger waiting time to shorter waiting time, by this we can reduce backlogging. For this we have to improve production, logistics and stock in our model. The first model for deteriorating items was formulated by Ghare and Schrader (1963). Chang \& Dye (1999) deliberated a decaying inventory model with time varying demand and partial backlogging. An optimal replenishment policy for noninstantaneous deteriorating items with stock-dependent demand was introduced by $\mathrm{Wu}$ et. al.(2006).Other inspiring articles related to this research area are Singh \& Singh (2008), Sugapriya \& Jeyaraman (2008),Dye (2013), Shukla et. al. (2013), Singh \& Sharma (2014), Jaggi (2014), Tayal et.al (2015) and Jaggi et. al.(2015), Khurana (2015) etc.

Learning phenomena cannot be ommit. By monitoring demand, shortage, holding cost $\&$ backlogged we can improve results as well as performance, shortage cannot be vanish. While developing inventory model we have to improve effective tools and machinery, production procedure, human resource environment. Learning, shortage and backlogging, we have developed a new effective inventory model. The learning phenomena defined by Wright [1936]. Jordan [1958] formulated that how to use the learning curve. An EPQ model under learning effect was developed by Fisk and Ballou [1985]. Balkhi [2003] analyzed an optimal production lot size for deteriorating items with learning effect. An inventory model for imperfect quality items with learning was introduced by Jaber et. al.(2008). Kumar et al. (2013) investigated a Learning effect on an inventory model with two-level storage and partial backlogging. An imperfect quality items with learning under two limited storage capacity was developed by Singh et.al.(2013). Singhal \& Singh (2015) proposed an inventory system with multi variate demand under volume flexibility and learning.

In this article a decaying inventory model with variable deterioration and stock dependent demand rate is developed. This model is explained both crisp and fuzzy environment. 
Shortages are permitted and partial backlogging. Learning effect is also considered in this model. Therefore, an inventory model with partial-backlogging is considered in fuzzy sense. The signed distance method is applied for defuzzification

\section{ASSUMPTIONS AND NOTATIONS:}

\subsection{Assumptions}

The basic assumptions are applied to analyze this inventory model:

1. The demand rate for the product is assumed to be stock dependent which is given by $D(t)=a+m I(t), I(t)>0$ and

$a, I(t)<0$, where $a>0,0<m<1$, $a>m$

2. The deteriorating rate $\theta(0<\theta<1)$ is time dependent.

3. Shortages are allowed and partial backlogged and backlogging rate is considered to be $B(t)=e^{-\delta(T-t)}, 0<\delta<1$.

4. Holding cost is partly constant and partly decreasing in each cycle due to learning effect of employees and is of the form $\left(h+\frac{h_{0}}{n^{\beta}}\right), \beta>0$

5. $t_{d}$ is the length of time in which the product has no deterioration.

6. $\mathrm{T}$ is the length of the cycle.

7. Lead-time is zero.

\subsection{Notations}

The basic notations are used to develop this inventory model:

1. A is the ordering cost.

2. $\mathrm{P}$ is the Purchasing cost.

3. $C_{1}$ is the Deterioration Cost.

4. $C_{2}$ is the Shortage Cost.

5. $C_{3}$ is the Lost Sale Cost.

6. No. of shipments is $\mathrm{n}$.

7. $\tilde{\theta}$ is the fuzzy deterioration rate.

8. $\tilde{A}$ is the fuzzy ordering cost.

9. $\tilde{C}_{1}$ is the fuzzy deterioration cost.

10. $\tilde{C}_{2}$ is the fuzzy shortage cost.

11. The Inventory Level at time $\mathrm{t}$ is $I_{1}(t)$ with $t \in\left[0, t_{d}\right]$
12. The Inventory Level at time $\mathrm{t}$ is $I_{2}(t)$ with $t \in\left[t_{d}, t_{1}\right]$

13. The Inventory Level at time $\mathrm{t}$ is $I_{3}(t)$ with $t \in\left[t_{1}, T\right]$

14. $T C\left(t_{1}, T\right)$ is the total cost of the system for crisp model.

15. $T \tilde{C}\left(t_{1}, T\right)$ is the total cost of the system for fuzzy model.

\section{MATHEMATICAL FORMULATION OF THE MODEL}

\section{Crisp model:}

Let $\mathrm{I}(\mathrm{t})$ be the inventory level at time $\mathrm{t}(0 \leq t \leq T)$. During the time interval $\left(0, t_{d}\right)$ the inventory level is depletes only owing to stock-dependent demand rate. The differential equation during the interval $\left(0, t_{d}\right)$ is given by

$$
\frac{d I_{1}(t)}{d t}=-\left[a+m I_{1}(t)\right], \quad 0 \leq t \leq t_{d}
$$

Again during the time interval $\left(t_{d}, t_{1}\right)$ inventory is dropping to zero due to demand rate and deterioration both. The differential equation during the interval $\left(t_{d}, t_{1}\right)$ is given by

$\frac{d I_{2}(t)}{d t}+\theta t I_{2}(t)=-\left[a+m I_{2}(t)\right], t_{d} \leq t \leq t_{1}$

After that during the time interval $\left(t_{1}, T\right)$ shortage starts and due to partial backlogging some sales are lost. The differential equation during the interval $\left(t_{1}, T\right)$ is given by

$$
\frac{d I_{3}(t)}{d t}=-a e^{-\delta(T-t)}, t_{1} \leq t \leq T
$$

With boundary conditions

$$
I_{1}(0)=I_{\text {ma.x. }}, I_{2}\left(t_{1}\right)=0 \& I_{3}\left(t_{1}\right)=0
$$

Solutions of these equations are

$$
\begin{aligned}
& I_{1}(t)=e^{-m t}\left[I_{m a . x .}-a\left(t+\frac{m t^{2}}{2}\right)\right] \\
& I_{2}(t)=a\left(e^{-\left(\frac{\theta^{2}}{2}+m t\right)}\right)\left[\left(t_{1}-t\right)+\frac{\theta}{6}\left(t_{1}^{3}-t^{3}\right)+\frac{m}{2}\left(t_{1}^{2}-t^{2}\right)\right]
\end{aligned}
$$




$$
I_{3}(t)=-a\left[t-\delta\left(T t-\frac{t^{2}}{2}\right)\right]+a\left[t_{1}-\delta\left(T t_{1}-\frac{t_{1}^{2}}{2}\right)\right]
$$

Considering the continuity at $t=t_{d}$ it follows from equation (5) \& (6) such that $I_{1}\left(t_{d}\right)=I_{2}\left(t_{d}\right)$

$e^{-m t_{d}} I_{\text {ma.x. }}-e^{-m t_{d}} a\left(t_{d}+\frac{m t_{d}^{2}}{2}\right)=$

$a\left(e^{-\left(\frac{\theta t_{d}^{2}}{2}+m t_{d}\right)}\right)\left[\left(t_{1}-t_{d}\right)+\frac{\theta}{6}\left(t_{1}^{3}-t_{d}^{3}\right)+\frac{m}{2}\left(t_{1}^{2}-t_{d}^{2}\right)\right]$

Now Maximum inventory level for each cycle is

$Q_{1}=I_{\text {ma.x. }}=a t_{d}\left(1+\frac{m}{2} t_{d}\right)+a e^{m t_{d}}\left[\left(t_{1}-t_{d}\right)+\frac{\theta}{6}\left(t_{1}^{3}-t_{d}^{3}\right)\right.$

$+\frac{m}{2}\left(t_{1}^{2}-t_{d}^{2}\right)+\frac{\theta}{2} t_{d}^{2}\left(t_{d}-t_{1}\right)+\frac{\theta m}{4} t_{d}^{2}\left(t_{d}^{2}-t_{1}^{2}\right)$

$\left.+m t_{d}\left(t_{1}-t_{d}\right)+\frac{\theta m}{6} t_{d}\left(t_{1}^{3}-t_{d}^{3}\right)\right]$

Now Equation (4) becomes

$I_{1}(t)=a t_{d}\left(1+\frac{m}{2} t_{d}\right) e^{-m t}-a t\left(1+\frac{m}{2} t\right) e^{-m t}+a e^{m\left(t_{d}-t\right)}$

$\left[\left(t_{1}-t_{d}\right)+\frac{\theta}{6}\left(t_{1}^{3}-t_{d}^{3}\right)+\frac{m}{2}\left(t_{1}^{2}-t_{d}^{2}\right)+\frac{\theta}{2} t_{d}^{2}\left(t_{d}-t_{1}\right)\right.$

$\left.+\frac{\theta m}{4} t_{d}^{2}\left(t_{d}^{2}-t_{1}^{2}\right)+m t_{d}\left(t_{1}-t_{d}\right)+\frac{\theta m}{6} t_{d}\left(t_{1}^{3}-t_{d}^{3}\right)\right]$

Put $t=T$ in equation (7) we get max. amount of backlogged per cycle as follows

$\left.Q_{2}=-I_{3}(T)=-a\left[T-\delta \frac{T^{2}}{2}\right)\right]+a\left[t_{1}-\delta\left(T t_{1}-\frac{t_{1}^{2}}{2}\right)\right]$

The order quantity is

$\mathrm{Q}=a t_{d}\left(1+\frac{m}{2} t_{d}\right)-a\left[T-\delta \frac{T^{2}}{2}\right]+a\left[t_{1}-\delta\left(T t_{1}-\frac{t_{1}^{2}}{2}\right)\right]+$

$a e^{m t_{d}}\left[\left(t_{1}-t_{d}\right)+\frac{\theta}{6}\left(t_{1}^{3}-t_{d}{ }^{3}\right)+\frac{m}{2}\left(t_{1}^{2}-t_{d}^{2}\right)+\frac{\theta}{2} t_{d}^{2}\left(t_{d}-t_{1}\right)\right.$

$\left.+\frac{\theta m}{4} t_{d}^{2}\left(t_{d}^{2}-t_{1}^{2}\right)+m t_{d}\left(t_{1}-t_{d}\right)+\frac{\theta m}{6} t_{d}\left(t_{1}^{3}-t_{d}^{3}\right)\right]$
3.1 The Purchasing cost is given by

$P C=P Q=P\left(Q_{1}+Q_{2}\right)$

$\mathrm{PC}=\mathrm{P}\left[a t_{d}\left(1+\frac{m}{2} t_{d}\right)-a\left[T-\delta \frac{T^{2}}{2}\right]+a\left[t_{1}-\delta\left(T t_{1}-\frac{t_{1}^{2}}{2}\right)\right]+\right.$

$a e^{m t_{d}}\left\{\left(t_{1}-t_{d}\right)+\frac{\theta}{6}\left(t_{1}^{3}-t_{d}{ }^{3}\right)+\frac{m}{2}\left(t_{1}^{2}-t_{d}^{2}\right)+\frac{\theta}{2} t_{d}^{2}\left(t_{d}-t_{1}\right)\right.$

$\left.\left.+\frac{\theta m}{4} t_{d}{ }^{2}\left(t_{d}^{2}-t_{1}^{2}\right)+m t_{d}\left(t_{1}-t_{d}\right)+\frac{\theta m}{6} t_{d}\left(t_{1}^{3}-t_{d}^{3}\right)\right\}\right]$

3.2 Ordering Cost is given by

$$
\mathrm{OC}=\mathrm{A}
$$

3.3 Holding cost is given by

$$
\begin{aligned}
& H C=\left(h+\frac{h_{0}}{n^{\alpha_{2}}}\right)\left[\int_{0}^{t_{d}} I_{1}(t) d t+\int_{t_{d}}^{t_{1}} I_{2}(t) d t\right] \\
& H C=\left(h+\frac{h_{0}}{n^{\alpha_{2}}}\right)\left[\int_{0}^{t_{d}}\left(a t_{d}\left(1+\frac{m}{2} t_{d}\right) e^{-m t}-a t\left(1+\frac{m}{2} t\right) e^{-m t}\right)+\right. \\
& \left(a e ^ { m ( t _ { d } - t ) } \left(\left(t_{1}-t_{d}\right)+\frac{\theta}{6}\left(t_{1}^{3}-t_{d}{ }^{3}\right)+\frac{m}{2}\left(t_{1}^{2}-t_{d}{ }^{2}\right)+\frac{\theta}{2} t_{d}{ }^{2}\left(t_{d}-t_{1}\right)\right.\right. \\
& \left.\left.+\frac{\theta m}{4} t_{d}{ }^{2}\left(t_{d}{ }^{2}-t_{1}^{2}\right)+m t_{d}\left(t_{1}-t_{d}\right)+\frac{\theta m}{6} t_{d}\left(t_{1}^{3}-t_{d}^{3}\right)\right)\right) d t+ \\
& \left.\int_{t_{d}}^{t_{1}}\left(a e^{-\left(\frac{\theta^{2}}{2}+m t\right)}\left(\left(t_{1}-t\right)+\frac{\theta}{6}\left(t_{1}^{3}-t^{3}\right)+\frac{m}{2}\left(t_{1}^{2}-t^{2}\right)\right)\right) d t\right]
\end{aligned}
$$

3.4 Deterioration Cost is given by

$$
\begin{aligned}
& D C=I_{2}\left(t_{d}\right)-\int_{t_{d}}^{t_{1}}\left(a+m I_{2}(t)\right) d t \\
& D C=I_{2}\left(t_{d}\right)-\int_{t_{d}}^{t_{1}}\left(a+m a e^{-\left(\frac{\theta t^{2}}{2}+m t\right)}\left(\left(t_{1}-t\right)+\frac{\theta}{6}\left(t_{1}^{3}-t^{3}\right)\right.\right.
\end{aligned}
$$$$
\left.\left.+\frac{m}{2}\left(t_{1}^{2}-t^{2}\right)\right)\right) d t
$$

3.5 Shortage cost is given by $S C=-C_{2} \int_{t_{1}}^{T} I_{3}(t) d t$ $S C=-C_{2} \int_{t_{1}}^{T}\left[-a\left(t-\delta\left(T t-\frac{t^{2}}{2}\right)\right)+a\left(t_{1}-\delta\left(T t_{1}-\frac{t_{1}^{2}}{2}\right)\right)\right] d t$

3.6 Lost Sales Cost due to lost sales is given by

$$
\begin{aligned}
& L S=C_{3} \int_{t_{1}}^{T} a\left(1-e^{-\delta(T-t)}\right) d t \\
& L S=C_{3} \frac{a \delta}{2}\left(T-t_{1}\right)^{2}
\end{aligned}
$$


3.7 Therefore, the total relevant inventory cost per unit time is given by (Crisp Model)

$$
T C\left(t_{1}, T\right)=\frac{1}{T}[D C+O C+P C+H C+S C+L S]
$$

\section{OPTIMAL SOLUTION PROCEDURE}

The total values of $\mathrm{t} 1$ and $\mathrm{T}$ which minimize total cost $\mathrm{TC}$ can be solved by differentiating equation (21), put

$$
\frac{\partial T C\left(t_{1}, T\right)}{\partial t_{1}}=0, \frac{\partial T C\left(t_{1}, T\right)}{\partial T}=0
$$

Provided it satisfies the condition

$$
\begin{aligned}
& \frac{\partial^{2} T C\left(t_{1}, T\right)}{\partial t_{1}^{2}}>0, \frac{\partial^{2} T C\left(t_{1}, T\right)}{\partial T^{2}}>0 \\
& {\left[\frac{\partial^{2} T C\left(t_{1}, T\right)}{\partial t_{1}^{2}}\right]\left[\frac{\partial^{2} T C\left(t_{1}, T\right)}{\partial T^{2}}\right]-\left[\frac{\partial^{2} T C\left(t_{1}, T\right)}{\partial T \partial t_{1}}\right]^{2}>0}
\end{aligned}
$$

\section{NUMERICAL EXPERIMENT}

Let us consider

$A=150, \theta=0.02, P=4, C_{1}=1.4, a=150$,

$m=0.44, \delta=0.01, C_{2}=2.5, C_{3}=2, h=5$,

$h_{0}=2, n=3, \beta=0.1, t_{d}=1.2$

Based on these input data, we get

$t_{1}=2.2718, \mathrm{~T}=5.42421$ and optimal total cost will be

$$
T C\left(t_{1}, T\right)=1494.51
$$

\section{SENSITIVITY ANALYSIS}

Sensitivity analysis is performed by changing the parameters and considering one parameter at a time, keeping the left over parameters at their original values

Table-(1) Variation in Deterioration cost parameter ' $C_{1}$,

\begin{tabular}{cccc}
\hline$C_{1}$ & $t_{1}$ & $T$ & TC \\
\hline 1.41 & 2.27404 & 5.44213 & 1495.21 \\
\hline 1.42 & 2.27458 & 5.44437 & 1495.98 \\
\hline 1.43 & 2.27505 & 5.4466 & 1496.63 \\
\hline 1.44 & 2.27555 & 5.44896 & 1497.31 \\
\hline
\end{tabular}

Table-(2) Variation in Deterioration rate ' $\theta$,

\begin{tabular}{cccc}
\hline$\theta$ & $t_{1}$ & $T$ & TC \\
\hline 0.03 & 2.23729 & 5.29071 & 1470.43 \\
\hline 0.04 & 2.2062 & 5.18694 & 1446.02 \\
\hline 0.05 & 2.17894 & 5.09124 & 1425.53 \\
\hline 0.06 & 2.15594 & 5.04701 & 1402.01 \\
\hline
\end{tabular}

Table-(3) Variation in shortage cost parameter ' $C_{2}$,

\begin{tabular}{cccc}
\hline$C_{2}$ & $t_{1}$ & $T$ & TC \\
\hline 2.6 & 2.27366 & 5.40955 & 1513.2 \\
\hline 2.7 & 2.2709 & 5.29606 & 1541.27 \\
\hline 2.8 & 2.26871 & 5.26503 & 1555.86 \\
\hline 2.9 & 2.271 & 5.24598 & 1575.13 \\
\hline
\end{tabular}

\section{OBSERVATIONS}

- From Table 1, If we increase the deterioration cost parameter ' $C_{1}$ 'then total cost TC increases.

- From Table 2, If we increase the deterioration rate $\theta$ 'then total cost TC decreases.

- From Table 3, If we increase the shortage cost parameter $C_{2}$ then the total cost TC increases.

\section{FUZZY-MATHEMATICAL MODEL}

Let us consider in this inventory model $\theta, A, C_{1}, a n d C_{2}$ as triangular fuzzy numbers, i.e. $\tilde{\theta}, \tilde{A}, \tilde{C}_{1}, \operatorname{and} \tilde{C}_{2}$. Let us assume that parameters $\tilde{\theta}, \tilde{A}, \tilde{C}_{1}$, and $\tilde{C}_{2}$ may change within some limits.

$\tilde{\theta}=\left(\theta-\Delta_{1}, \theta, \theta+\Delta_{2}\right)$, where $0<\Delta_{1}<\theta \quad$ and

$\Delta_{1} \Delta_{2}>0$

$\tilde{A}=\left(A-\Delta_{3}, A, A+\Delta_{4}\right)$, where $0<\Delta_{3}<A$ and

$\Delta_{3} \Delta_{4}>0$,

$\tilde{C}_{1}=\left(C_{1}-\Delta_{5}, C_{1}, C_{1}+\Delta_{6}\right)$, where $0<\Delta_{5}<C_{1}$

and $\Delta_{5} \Delta_{6}>0$,

$\tilde{C}_{2}=\left(C_{2}-\Delta_{7}, C_{2}, C_{2}+\Delta_{8}\right)$, where $0<\Delta_{7}<C_{2}$ and $\Delta_{7} \Delta_{8}>0$,

By signed distance Method

$$
\begin{aligned}
& \tilde{\theta}=\theta+\frac{1}{4}\left(\Delta_{2}-\Delta_{1}\right) \\
& \tilde{A}=A+\frac{1}{4}\left(\Delta_{4}-\Delta_{3}\right) \\
& \tilde{C}_{1}=C_{1}+\frac{1}{4}\left(\Delta_{6}-\Delta_{5}\right) \\
& \tilde{C}_{2}=C_{2}+\frac{1}{4}\left(\Delta_{8}-\Delta_{7}\right)
\end{aligned}
$$


Now defuzzified total cost per unit time by signed distance method is given by

$T \tilde{C}\left(t_{1}, T\right)=\frac{1}{4}\left[T \tilde{C} F_{1}\left(t_{1}, T\right)+2 T \tilde{C} F_{2}\left(t_{1}, T\right)+T \tilde{C} F_{3}\left(t_{1}, T\right)\right](30)$

The necessary condition for minimize total cost are

$\frac{\partial T \tilde{C}\left(t_{1}, T\right)}{\partial t_{1}}=0, \frac{\partial T \tilde{C}\left(t_{1}, T\right)}{\partial T}=0$

Provided it satisfies the condition

$$
\begin{aligned}
& \frac{\partial^{2} T \tilde{C}\left(t_{1}, T\right)}{\partial t_{1}^{2}}>0, \frac{\partial^{2} T \tilde{C}\left(t_{1}, T\right)}{\partial T^{2}}>0 \\
& {\left[\frac{\partial^{2} T \tilde{C}\left(t_{1}, T\right)}{\partial t_{1}^{2}}\right]\left[\frac{\partial^{2} T \tilde{C}\left(t_{1}, T\right)}{\partial T^{2}}\right]-\left[\frac{\partial^{2} T \tilde{C}\left(t_{1}, T\right)}{\partial T \partial t_{1}}\right]^{2}>0}
\end{aligned}
$$

Using the software Mathematica, we get optimum value of $t_{1}^{*}, T^{*}$ and the optimum total cost $T \tilde{C}\left(t_{1}, T\right)$.

\section{FUZZY NUMERICAL EXAMPLE}

Let us consider

$$
\begin{aligned}
& \tilde{A}=(130,150,170), a=150, \tilde{C}_{1}=(1,1.4,1.8), t_{d}=1.2, \\
& C_{3}=2, h_{0}=2, \delta=0.01, \tilde{C}_{2}=(2.1,2.5,2.9), h=5, \\
& \tilde{\theta}=(0.01,0.02,0.03), m=0.44, P=4, n=3, \beta=0.1
\end{aligned}
$$

Based on these input data, we get

$t_{1}^{*}=2.1678, T^{*}=5.20747$ and total cost

$$
T \tilde{C}\left(t_{1}, T\right)=649.637 \text {. }
$$

\section{CONCLUSION}

This paper presents a crisp and fuzzy inventory model for non-instantaneous decaying items with shortages considering demand rate is time dependent. The demand, deterioration rate, ordering cost and shortage cost are represented by triangular fuzzy numbers. For defuzzification signed distance method is utilized to evaluate the optimal total cost. The proposed model is more practical due to the impreciseness in inventory costs and demand. Considering variables as fuzzy numbers is also more useful business strategy to cope up with ups and downs conditions of the market. To make inventory model more realistic, we also consider stock dependent demand and partial backlogging since all shortages cannot be fully backlogged. At the end numerical example and sensitivity analysis is elaborated. The whole calculation part is done using Mathematica. For the future research we can incorporate some other parameters of inventory control system.

\section{REFERENCES}

[1] Balkhi, Z.T.,(2003), 'The Effect of learning on the optimal production lot size for deteriorating and partially backordered items with time varying demand and deterioration rates', Applied Mathematical Modeling, 27,763-779.
[2] Chang, H.J., Dye, C.Y., (1999), 'An EOQ model for deteriorating items with time varying demand and partial backlogging', Journal of the Operational Research Society,50, 1176-1182.

[3] De, P.K., Rawat, A.,(2011), 'A Fuzzy Inventory Model without Shortages Using Triangular Fuzzy Number', Fuzzy Information and Engineering, 3, 59-68.

[4] Dubois, D., Prade, H., (1978), 'Operations on Fuzzy Numbers' International Journal of System Science, 9, 613-626.

[5] Dutta, D., Kumar, P., (2013), 'Optimal Ordering Policy for an Inventory Model for Deteriorating Items without Shortages by Considering Fuzziness in Demand Rate, Ordering Cost and Holding Cost', International Journal of Advanced Innovation and Research, 2, 320-325.

[6] Dye, C.Y.(2013), 'The effect of preservation technology investment on a non-instantaneous deteriorating inventory model' Omega, 41(5),872 - 880 .

[7] Fisk, J.C., Ballou, D.P., (1985), 'Production lot sizing under learning effect' AIIE Transactions, 17, 33-37.

[8] Ghare, P.M., Schrader, G. P.,(1963), 'A model for an exponentially decaying inventory, Journal of Industrial Engineering,14, (5),248-243.

[9] Halim,K.A,.Giri,B.C.\& Chaudhuri, K.S.(2008), 'Fuzzy economic order quantity model for perishable items with stochastic demand, partial backlogging and fuzzy deteriorating rate', International Journal of Operational Research, 3, 77-96.

[10] Jaber, M.Y., Goyal, S.K., Imran, M. (2008), 'Economic production quantity model for items with imperfect quality subject to learning effects', International Journal of Production Economics, 115,143- 150.

[11] Jaggi, C.K, Sharma, A., Tiwari .S. (2015), 'Credit financing in economic ordering policies for noninstantaneous deteriorating items with price dependent demand under permissible delay in payments' International Journal of Industrial Engineering Computations,(6), 481-502 .

[12] Jaggi, C.K., Pareek, S., Sharma, A., Nidhi. (2012), 'Fuzzy Inventory Model for Deteriorating Items with Time Varying Demand and Shortages', American Journal of Operational Research,2, 81-92.

[13] Jaggi, C.k.,(2014), 'An Optimal Replenishment Policy for Non Instantaneous Deteriorating Items with Price Dependent Demand and Time-Varying Holding Cost' International Scientific Journal on Science Engineering \& Technology, 17(03).

[14] Jain, R. (1976), 'Decision Making in the Presence of Fuzzy Variables' IIIE Transactions on Systems, Man and Cybernetics.17, 698-703.

[15] Jordan, R.B.(1958), 'Learning how to use the learning curve', N.A.A. Bull, 39 (5), 27-39.

[16] Kacpryzk, J., Staniewski, P. (1982), 'Long Term Inventory Policy Making through Fuzzy Decision Making Methods', Fuzzy Sets and System, 8, 117-132. 
[17] Kao, C.K., Hsu, W.K. (2002), 'A Single Period Inventory Model with Fuzzy Demand', Computers and Mathematics with Applications, 43, 841-848.

[18] Khurana, D.,(2015), 'Two Warehouse Inventory Model for Deteriorating Items with Time Dependent Demand under Inflation',International Journal of Computer Applications, 114,7, 0975 - 8887

[19] Kumar ,S., Rajput, U.S.(2015), 'Fuzzy Inventory Model for Deteriorating items with Time Dependent Demand and Partial Backlogging', Applied Mathematics, 6,496509.

[20] Kumar, N., Singh, S.R., Kumari, K. (2013-a), 'Learning effect on an inventory model with two-level storage and partial backlogging under inflation', International Journal of Services and Operations Management, 16 (1), $105-122$.

[21] Mahapatra, N.K., Maiti,M.(2006), 'A fuzzy stochastic approach to multi-objective inventory model of deteriorating items with various types of demand and time dependent holding cost', Journal of the Operational Research Society of India, 43 (2), 117-131.

[22] Saha, S., Chakrabarti, T.(2012), 'Fuzzy EOQ Model with Time Dependent Demand and Deterioration with Shortages', IOSR Journal of Mathematics, 2, 46-54.

[23] Shukla, H. S., Shukla, V., Yadav, S. K.(2013), 'EOQ model for deteriorating items with exponential demand rate and shortages' Uncertain Supply Chain Management, 1(2), 67-76.

[24] Singh, S., Jain, S., Pareek, S. (2103), 'An imperfect quality items with learning and inflation under two limited storage capacity', International Journal of Industrial Engineering Computations, 4 (4), 479-490.

[25] Singh, S.R., Sharma, S. (2014), 'Optimal trade-credit policy for perishable items deeming imperfect production and stock dependent demand', International Journal of Industrial Engineering Computations. 5(1), 151-168. under inflation in a supply chain' International Review of Pure and Advanced Mathematics, 1, 31-39.

[27] Singhal, S.,Singh, S. R.(2015), 'Modelling of an inventory system with multi variate demand under volume flexibility and learning' Uncertain Supply Chain Management, 3(2), 147-158.

[28] Sugapriya C., Jeyaraman K. (2008), 'An EPQ model for non-instantaneous deteriorating item in which holding cost varies with time' Electronic Journal of Applied Statistical Analysis.1, (1),16-23.

[29] Tayal , S., Singh, S.R., Sharma, R., Singh, A. P.(2015), 'An EPQ model for non-instantaneous deteriorating item with time dependent holding cost and exponential demand rate', International Journal of Operational Research, 23,(2),145-161.

[30] Wright, T. (1936), 'Factors affecting the cost of airplanes' Journal of Aeronautical Science, 3( 4),122128(1936).

[31] Wu, K.S, Ouyang, L.Y., Yang, C.T.(2006), ‘An optimal replenishment policy for non-instantaneous deteriorating items with stock-dependent demand and partial backlogging' Int. J. Production Economics 101,369-384.

[32] Yao, J.S., Chiang, J.(2003), 'Inventory without backorder with fuzzy total cost and fuzzy storing cost defuzzified by centroid and signed distance' European Journal of Operational Research, 148, 401-409.

[33] Yao, J.S., Lee, H. M.(1996), 'Fuzzy inventory with backorder for fuzzy order quantity' Information Sciences, 93, 283-319.

[34] Zadeh, L.A.(1965), 'Fuzzy Set' Information Control, 8,338-353.

[35] Zimmerman, H.J. (1983), 'Using Fuzzy Sets in Operational Research' European Journal of Operation Research, 13, 201-206.

[26] Singh, S.R., Singh, C.(2008), 'Optimal ordering policy for decaying items with stock dependent demand

\section{APPENDIX}

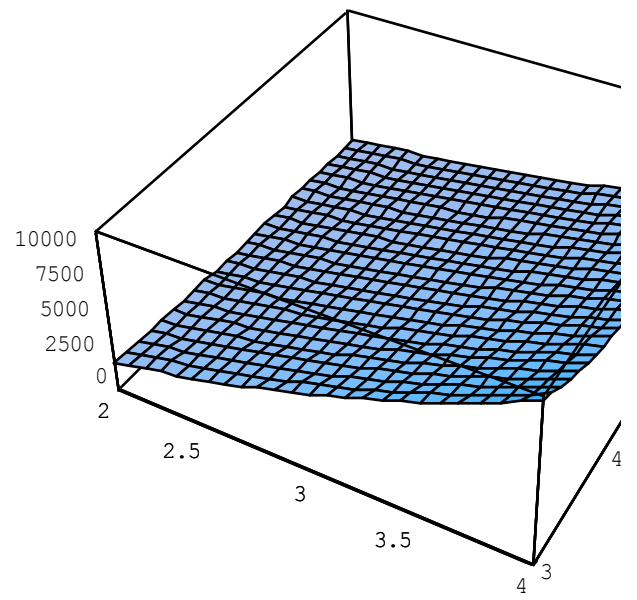

Fig. Convexity of the total cost function (Crisp Model) 
Total cost function for fuzzy model from equation no.(30)

Where

$$
\begin{aligned}
& T \tilde{C} F_{1}\left(t_{1}, T\right)=\frac{1}{T}\left[( C _ { 1 } - \Delta _ { 5 } ) \left\{\left(\frac{\left(\theta-\Delta_{1}\right)}{6}\right)\left(t_{1}^{3}-t_{d}^{3}\right)\left(1+m\left(t_{1}+t_{d}\right)\right)+\left(t_{d}-t_{1}\right)\left\{\frac{\left(\theta-\Delta_{1}\right)}{2} t_{d}^{2}+m\left(t_{1}-t_{d}\right)\right.\right.\right. \\
& \left.\left.+\frac{m\left(\theta-\Delta_{1}\right)}{6} t_{1}^{3}\right\}+m\left(t_{1}^{2}-t_{d}^{2}\right)\left(1-\frac{\left(\theta-\Delta_{1}\right)}{4} t_{d}^{2}\right)-\frac{\left(\theta-\Delta_{1}\right) m}{12}\left(t_{1}^{4}-t_{d}^{4}\right)\right\}+\left(A-\Delta_{3}\right)+\mathrm{P}\left\{a e^{m t_{d}}\right. \\
& \left\{\left(t_{1}-t_{d}\right)+\frac{\left(\theta-\Delta_{1}\right)}{6}\left(t_{1}^{3}-t_{d}^{3}\right)+\frac{m}{2}\left(t_{1}^{2}-t_{d}^{2}\right)+\frac{\left(\theta-\Delta_{1}\right)}{2} t_{d}^{2}\left(t_{d}-t_{1}\right)+\frac{\left(\theta-\Delta_{1}\right) m}{4} t_{d}^{2}\left(t_{d}^{2}-t_{1}^{2}\right)+m t_{d}\left(t_{1}-t_{d}\right)\right. \\
& \left.\left.\left.+\frac{\left(\theta-\Delta_{1}\right) m}{6} t_{d}\left(t_{1}^{3}-t_{d}^{3}\right)\right\}+a\left\{t_{d}\left(1+\frac{m}{2} t_{d}\right)-\left(T-\delta \frac{T^{2}}{2}\right)\right)+\left(t_{1}-\delta\left(T t_{1}-\frac{t_{1}^{2}}{2}\right)\right)\right\}\right\} \\
& +a\left(h+\frac{h_{0}}{n^{\alpha_{2}}}\right)\left\{\left(t_{d}+t_{d}^{2}\left(m-\frac{1}{2}\right)\left\{\left(t_{1}-t_{d}\right)\left(1-\frac{\left(\theta-\Delta_{1}\right)}{2} t_{d}^{2}+m t_{d}\right)+\left(\frac{\left(\theta-\Delta_{1}\right)}{6}\right)\left(t_{1}^{3}-t_{d}^{3}\right)\left(1+m t_{d}\right)\right.\right.\right. \\
& \left.+\frac{m}{2}\left(t_{1}^{2}-t_{d}^{2}\right)\left(1-\frac{\left(\theta-\Delta_{1}\right)}{2} t_{d}^{2}\right)\right\}+\frac{t_{d}^{2}}{6}\left(m t_{d}-3\right)+\left(t_{1}-t_{d}\right)\left(t_{1}+\frac{\left(\theta-\Delta_{1}\right)}{6} t_{1}^{3}+\frac{m}{2} t_{1}^{2}\right)+\left(\frac{m t_{1}}{2}+\frac{\left(\theta-\Delta_{1}\right) m t_{1}^{3}}{12}-\frac{1}{2}\right) \\
& \left.\left(t_{1}^{2}-t_{d}^{2}\right)-\left(t_{1}^{3}-t_{d}^{3}\right)\left(\frac{m}{2}+\frac{\left(\theta-\Delta_{1}\right) m t_{1}^{2}}{12}+\frac{\left(\theta-\Delta_{1}\right) t_{1}}{6}\right)+\frac{\left(\theta-\Delta_{1}\right)\left(t_{1}^{4}-t_{d}^{4}\right)}{12}+\frac{\left(\theta-\Delta_{1}\right) m\left(t_{1}^{5}-t_{d}^{5}\right)}{60}\right\}+C_{3} \frac{a \delta}{2}\left(T-t_{1}\right)^{2} \\
& -\left(C_{2}-\Delta_{7}\right) a\left\{\frac{-\delta\left(T^{3}-t_{1}^{3}\right)}{6}-\frac{(1-\delta T)\left(T^{2}-t_{1}^{2}\right)}{2}+\left(T-t_{1}\right)\left(t_{1}-\delta\left(T t_{1}-\frac{1}{2} t_{1}^{2}\right)\right\}\right.
\end{aligned}
$$$$
T C \tilde{F}_{2}\left(t_{1}, T\right)=\frac{1}{T}\left[C _ { 1 } \left\{\left(\frac{\theta}{6}\right)\left(t_{1}^{3}-t_{d}^{3}\right)\left(1+m\left(t_{1}+t_{d}\right)\right)+\left(t_{d}-t_{1}\right)\left(\frac{\theta}{2} t_{d}^{2}+m\left(t_{1}-t_{d}\right)+\frac{m \theta}{6} t_{1}^{3}\right)\right.\right.
$$$$
\left.+m\left(t_{1}^{2}-t_{d}^{2}\right)\left(1-\frac{\theta}{4} t_{d}^{2}\right)-\frac{\theta m}{12}\left(t_{1}^{4}-t_{d}^{4}\right)\right\}+A+\mathrm{P}\left\{a e ^ { m t _ { d } } \left\{\left(t_{1}-t_{d}\right)+\frac{\theta}{6}\left(t_{1}^{3}-t_{d}^{3}\right)+\frac{m}{2}\left(t_{1}^{2}-t_{d}^{2}\right)\right.\right.
$$$$
\left.+\frac{\theta}{2} t_{d}^{2}\left(t_{d}-t_{1}\right)+\frac{\theta m}{4} t_{d}^{2}\left(t_{d}^{2}-t_{1}^{2}\right)+m t_{d}\left(t_{1}-t_{d}\right)+\frac{\theta m}{6} t_{d}\left(t_{1}^{3}-t_{d}^{3}\right)\right\}+a t_{d}\left(1+\frac{m}{2} t_{d}\right)
$$$$
\left.\left.-a\left[T-\delta \frac{T^{2}}{2}\right)\right]+a\left[t_{1}-\delta\left(T t_{1}-\frac{t_{1}^{2}}{2}\right)\right]\right\}+a\left(h+\frac{h_{0}}{n^{\alpha_{2}}}\right)\left\{\left(t_{d}+t_{d}^{2}\left(m-\frac{1}{2}\right)\left\{\left(t_{1}-t_{d}\right)\left(1-\frac{\theta}{2} t_{d}^{2}+m t_{d}\right)\right.\right.\right.
$$$$
\left.+\left(\frac{\theta}{6}\right)\left(t_{1}^{3}-t_{d}^{3}\right)\left(1+m t_{d}\right)+\frac{m}{2}\left(t_{1}^{2}-t_{d}^{2}\right)\left(1-\frac{\theta}{2} t_{d}^{2}\right)\right\}+\frac{t_{d}^{2}}{6}\left(m t_{d}-3\right)+\left(t_{1}-t_{d}\right)\left(t_{1}+\frac{\theta}{6} t_{1}^{3}+\frac{m}{2} t_{1}^{2}\right)
$$$$
\left.+\left(t_{1}^{2}-t_{d}^{2}\right)\left(\frac{m t_{1}}{2}+\frac{\theta m t_{1}^{3}}{12}-\frac{1}{2}\right)-\left(t_{1}^{3}-t_{d}^{3}\right)\left(\frac{m}{2}+\frac{\theta m t_{1}^{2}}{12}+\frac{\theta t_{1}}{6}\right)+\frac{\theta\left(t_{1}^{4}-t_{d}^{4}\right)}{12}+\frac{\theta m\left(t_{1}^{5}-t_{d}^{5}\right)}{60}\right\}
$$ 


$$
\begin{aligned}
& +C_{3} \frac{a \delta}{2}\left(T-t_{1}\right)^{2}-C_{2} a\left\{\frac{-\delta\left(T^{3}-t_{1}^{3}\right)}{6}-\frac{(1-\delta T)\left(T^{2}-t_{1}^{2}\right)}{2}+\left(T-t_{1}\right)\left(t_{1}-\delta\left(T t_{1}-\frac{1}{2} t_{1}^{2}\right)\right\}\right] \\
& T C \tilde{F}_{3}\left(t_{1}, T\right)=\frac{1}{T}\left[( C _ { 1 } + \Delta _ { 6 } ) \left\{\left(\frac{\left(\theta-\Delta_{2}\right)}{6}\right)\left(t_{1}^{3}-t_{d}^{3}\right)\left(1+m\left(t_{1}+t_{d}\right)\right)+\left(t_{d}-t_{1}\right)\left(\frac{\left(\theta-\Delta_{2}\right)}{2} t_{d}^{2}\right.\right.\right. \\
& \left.\left.+m\left(t_{1}-t_{d}\right)+\frac{m\left(\theta-\Delta_{2}\right)}{6} t_{1}^{3}\right)+m\left(t_{1}^{2}-t_{d}^{2}\right)\left(1-\frac{\left(\theta-\Delta_{2}\right)}{4} t_{d}^{2}\right)-\frac{\left(\theta-\Delta_{2}\right) m}{12}\left(t_{1}^{4}-t_{d}^{4}\right)\right\}+\left(A+\Delta_{4}\right) \\
& +C_{3} \frac{a \delta}{2}\left(T-t_{1}\right)^{2}+\mathrm{P}\left\{a e ^ { m t _ { d } } \left\{\left(t_{1}-t_{d}\right)+\frac{\left(\theta+\Delta_{2}\right)}{6}\left(t_{1}^{3}-t_{d}^{3}\right)+\frac{m}{2}\left(t_{1}^{2}-t_{d}{ }^{2}\right)+\frac{\left(\theta+\Delta_{2}\right)}{2} t_{d}{ }^{2}\left(t_{d}-t_{1}\right)\right.\right. \\
& \left.+m t_{d}\left(t_{1}-t_{d}\right)+\frac{\left(\theta+\Delta_{2}\right) m}{4} t_{d}^{2}\left(t_{d}^{2}-t_{1}^{2}\right)+\frac{\left(\theta+\Delta_{2}\right) m}{6} t_{d}\left(t_{1}^{3}-t_{d}^{3}\right)\right\}+a\left\{\left(t_{1}-\delta\left(T t_{1}-\frac{t_{1}^{2}}{2}\right)\right)+\right. \\
& \left.\left.t_{d}\left(1+\frac{m}{2} t_{d}\right)-\left(T-\delta \frac{T^{2}}{2}\right)\right\}\right\}+a\left(h+\frac{h_{0}}{n^{\alpha_{2}}}\right)\left\{\left(t_{d}+t_{d}^{2}\left(m-\frac{1}{2}\right)\left\{\left(1-\frac{\left(\theta+\Delta_{2}\right)}{2} t_{d}^{2}+m t_{d}\right)\left(t_{1}-t_{d}\right)\right.\right.\right. \\
& \left.+\left(\frac{\left(\theta+\Delta_{2}\right)}{6}\right)\left(t_{1}^{3}-t_{d}^{3}\right)\left(1+m t_{d}\right)+\frac{m}{2}\left(t_{1}^{2}-t_{d}^{2}\right)\left(1-\frac{\left(\theta+\Delta_{2}\right)}{2} t_{d}^{2}\right)\right\}+\left(t_{1}+\frac{\left(\theta+\Delta_{2}\right)}{6} t_{1}^{3}+\frac{m}{2} t_{1}^{2}\right)\left(t_{1}-t_{d}\right) \\
& +\frac{t_{d}^{2}}{6}\left(m t_{d}-3\right)+\left(t_{1}^{2}-t_{d}^{2}\right)\left(\frac{m t_{1}}{2}+\frac{\left(\theta+\Delta_{2}\right) m t_{1}^{3}}{12}-\frac{1}{2}\right)-\left(t_{1}^{3}-t_{d}^{3}\right)\left(\frac{m}{2}+\left(\theta+\Delta_{2}\right)\left(\frac{m t_{1}^{2}}{12}+\frac{t_{1}}{6}\right)\right)+\left(\theta+\Delta_{2}\right) \\
& \left.\left(\frac{\left(t_{1}^{4}-t_{d}^{4}\right)}{12}+\frac{m\left(t_{1}^{5}-t_{d}^{5}\right)}{60}\right)\right\}-C_{2} a\left\{\frac{-\delta\left(T^{3}-t_{1}^{3}\right)}{6}-\frac{(1-\delta T)\left(T^{2}-t_{1}^{2}\right)}{2}+\left(T-t_{1}\right)\left(t_{1}-\delta\left(T t_{1}-\frac{1}{2} t_{1}^{2}\right)\right\}\right]
\end{aligned}
$$

